\title{
EXPERIMENTS ON BEAM-BEAM DEPOLARIZATION AT LEP
}

\author{
R. Assmann ${ }^{\star}$, A. Blondel ${ }^{\star}$, B. Dehning, A. Drees ${ }^{\circ}$, P. Grosse-Wiesmann, H. Grote, M. Placidi, R. Schmidt, \\ F. Tecker ${ }^{\dagger}$, J. Wenninger
}

\author{
CERN, CH-1211 Geneva 23, Switzerland \\ * SLAC, Stanford Univ., P.O.Box 4349, CA 94309, USA \\ * Ecole Polytechnique, Paris, France \\ - Universität Wuppertal, Germany \\ † Physikalisches Institut III A, RWTH Aachen, Germany
}

Abstract

The possibility of obtaining transverse polarization with colliding beams was studied at LEP during 1994. A level of transverse beam polarization of $40 \%$ was achieved with $\mathrm{e}^{+} \mathrm{e}^{-}$collisions in one of the experimental interaction points. the collision of one electron and one positron bunch was $1.5 \times 10^{30} \mathrm{~cm}^{-2} \mathrm{~s}^{-1}$. The polarization level was not limited by the beam-beam interaction: from three circulating electron bunches only one was colliding with one positron bunch, whereas the beams were separated by electrostatic de $\mathrm{e}^{-}$ectors at the interaction points of the other electron bunches. No signi®cant difference between the polarization level of colliding and non colliding bunches was noticed. Betatron- and synchrotron frequencies as well as spin tune were chosen in order to avoid beam-beam driven depolarizing resonances.

\section{INTRODUCTION}

During 1994 a series of experiments were performed to assess the possibility of sustaining polarization with colliding beams at LEP. Polarization in collision would allow beam energy measurement during luminosity ${ }^{\circledR} 11 \mathrm{~s}$ and future experiments using colliding polarized beams.

To precisely determine mass and width of the $\mathrm{Z}$ boson a calibration of the beam energy by resonant depolarization is required. The calibration is performed at the end of a luminosity (Bll with non colliding beams [1]. A transverse polarization level of at least $10 \%$ with beams in collision would allow to reduce the time required for the energy calibration. The installation of a spin rotator at LEP, although not foreseen for the next years, would allow to collide longitudinal polarized beams and to measure the weak mixing angle $\theta_{w}$ with an accuracy which can not be achieved by other methods. Such an experiment requires a beam polarization of at least $50 \%$ [2].

\section{MAXIMUM POLARIZATION FOR A SINGLE BEAM}

A maximum level of transverse polarization for a single beam of $(57 \pm 3 \%)$ has been achieved using various techniques to compensate depolarizing effects [3] :

- the vertical closed orbit is carefully corrected to a rms value of 0.4-0.5 mm.

- the spin rotation due to the experimental solenoid magnetic ®eld of up to $66 \mathrm{mrad} / \mathrm{turn}$ which reduces the polarization level to less than $5 \%$ is compensated by a proper con®guration of vertical closed orbit bumps of both sides of the solenoids [4]

- the beam energy is selected for the spin tune to be close to $\gamma a=n+0.5$, with $\mathrm{n}$ an integer number, in order to minimize the depolarizing effects from integer resonances $(\gamma$ is the gamma-factor for the electrons, and $a$ is the anomalous magnetic momentum).

- Harmonic spin matching is applied to minimize the spurious tilt of the spin precession axis by compensating those harmonics of the Fourier expansion of the closed orbit which lead to a depolarization [5]. A novel implementation of the harmonic spin matching was developed for LEP by deriving the amplitude of the correcting bumps for the harmonics of interest directly from the beam position measurements [6].

\section{BEAM-BEAM EFFECTS}

The beam dynamics is strongly affected by beam-beam effects, as one beam acts on the counterrotating particles as a strong nonlinear lens. The strength of the beam-beam effect is characterized by the linear beam-beam tune shift. Beam-beam effects limit the maximum tune shift to a value of about 0.04 per interaction point for e+e- storage rings. If the operating parameters such as betatron and synchrotron tunes are not carefully chosen, the beam-beam tune shift as well as the attainable luminosity is limited to much lower values. Beam-beam effects can lead to an emittance increase and to particle losses. It has also been observed, that the distribution of particle amplitudes, which is Gaussian for a single beam, develops tails in the presence of beam-beam effects [7].

The in ${ }^{-}$uence of the beam-beam interaction on the beam polarization is twofold :

- the particle trajectory is de ected by an angle $\alpha$ due to the interaction with the counterrotating beam. The spin vector rotates by an angle : $\gamma a \times \alpha$. This effect can cause higher order depolarizing resonances.

- the excitation of vertical betatron oscillations by beambeam effects at the beam-beam limit can increase the strength of depolarizing resonances, in particular of those resonances where the condition $\gamma a=Q_{y} \pm n$ is ful®lled, with $Q_{y}$ the vertical betatron tune.

At PETRA a decrease of the transverse polarization was observed with increased beam-beam tune shift. At the same time the vertical beam size increased [8].

The in ${ }^{-}$uence of the beam-beam interaction on the polarization if not fully understood. Currently both at HERA [9] and at LEP [10] the depolarization by beam-beam effects is experimentally investigated. 


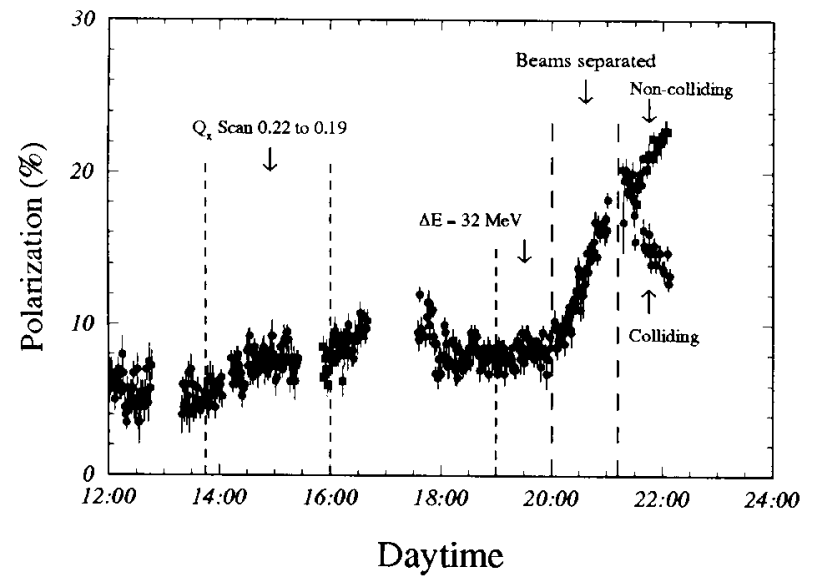

Figure. 1. Polarization level during ${ }^{\circledR r s t}$ experiment

\section{FIRST EXPERIMENT}

The ${ }^{\circledR}$ rst experiment performed at LEP demonstrated a strong depolarization due to the beam-beam interaction. 8 electron and 8 positron bunches were injected into LEP. The bunches were colliding at the four interaction points (IP) where LEP physics experiments are located, and separated at four other points in the vertical plane by electrostatic separators. To avoid collisions in the arcs additional separators are installed to separate the beams in the horizontal plane $\left({ }^{\circ}\right.$ Pretzel scheme $\left.{ }^{\circ}\right)$ [11]. Even with eight bunches per beam collisions can be avoided: For injection as well as for machine experiments the beams can be separated in the vertical plane at the collision points with LEP physics experiments.

The beam energy was $45.60 \mathrm{GeV}$, close to the optimum energy for the production of $\mathrm{Z}$ bosons. The betatron tunes were adjusted to $\mathrm{Q}_{x}=90.231$ and $\mathrm{Q}_{y}=76.153$. The linear beam-beam tune shift parameter was 0.015 . Only the polarization of the electron bunches was monitored. As show in $® \mathrm{~g}$. 1, the polarization level did not exceed $10 \%$ during the ${ }^{\circledR}$ rst part of the experiment when the beams were colliding at the IPs. An increase of the polarization level was observed when the horizontal betatron tune was changed to $\mathrm{Q}_{x}=90.187$. A scan of the beam energy from 45.602 $\mathrm{GeV}$ to $45.576 \mathrm{GeV}$ had no in ${ }^{-}$uence on the polarization level.

Later the beams were separated, and the polarization level increased. Four of the eight positron bunches were dumped and the vertical separation around the collision points with LEP physics experiments was switched off. This left four of the eight electron bunches colliding. For the noncolliding electron bunches the polarization kept increasing, whereas for the four colliding bunches the polarization level decreased.

\section{SECOND EXPERIMENT}

The second experiment was performed at the end of a luminosity ${ }^{\circledR} 11$ at a beam energy of about $45.60 \mathrm{GeV}$. For energy calibration the beams were separated at all four collision points and a polarization level of $20 \%$ was obtained. Then the beams were brought into collision at a single interaction point. The vertical electron beam size increased by about $10 \%$ with the beams

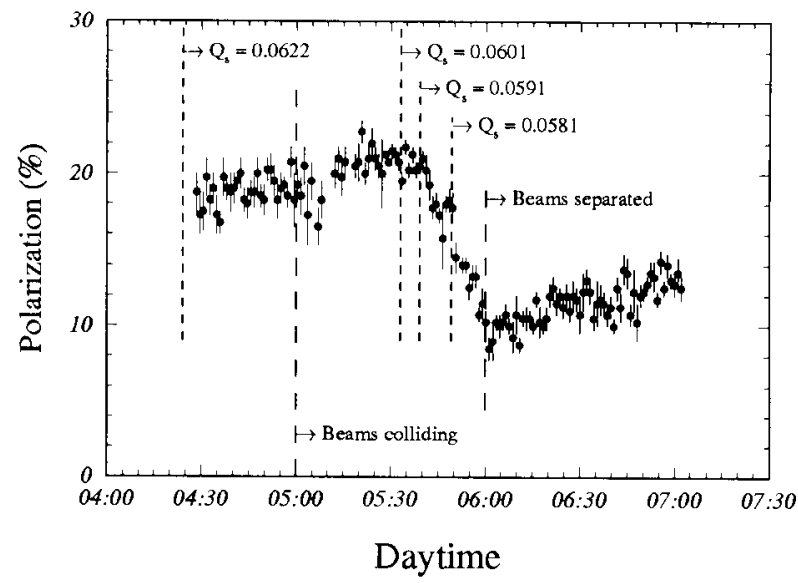

Figure. 2. Polarization level during second experiment

in collision, but no depolarization due to beam-beam effects was observed. The betatron tunes were $\mathrm{Q}_{x}=90.226$ and $\mathrm{Q}_{y}=$ 76.165 and the noninteger part of the spin tune $\gamma a=0.48$. After the beams were colliding, the luminosity was increased from $2.0 \times 10^{30} \mathrm{~cm}^{-2} \mathrm{~s}^{-1}$ to $2.4 \times 10^{30} \mathrm{~cm}^{-2} \mathrm{~s}^{-1}$ by an optimization of the beam overlap in the vertical plane with electrostatic separators installed left and right of the IPs. During this optimization no change of the polarization level was noticed. The linear beam-beam tune shift reached 0.017 .

To better understand the dependence of the beam-beam depolarization on the operating conditions the synchrotron tune was changed from 0.0622 to 0.0591 (see $® g .2$ ). The polarization level decreased to about $17 \%$. A further reduction of the synchrotron tune to 0.0581 lead to a strongly decreased polarization level of $10 \%$. When the beams were separated again (leaving the synchrotron tune at a value of 0.0581) the polarization started to rise, indicating that the depolarization was indeed caused by beam-beam effects.

\section{THIRD EXPERIMENT}

This experiment was performed at a beam energy of 44.71 $\mathrm{GeV}$. Three electron bunches and one positron bunch were injected. With such a scheme one electron bunch and one positron bunch collide in the interaction points 2 and 6 , if the separation bumps are turned off, whereas the other electron bunches meet the positron bunch at points where the beam are separated. This allows a direct comparison of the polarization levels of a colliding and a non colliding bunch.

The aim for this experiment was to achieve the highest possible polarization level with colliding beams. The highest polarization level of 57\% was achieved in 1993 with one beam, but during the energy calibrations in 1994 it never exceeded 25\% (the reasons for the low polarization level are not well understood, it is assumed that alignment of the magnets and precision of the beam orbit measurement system was slightly degraded during 1994).

All potential depolarizing sources were turned off :

- the solenoid magnets in the HEP experiments 


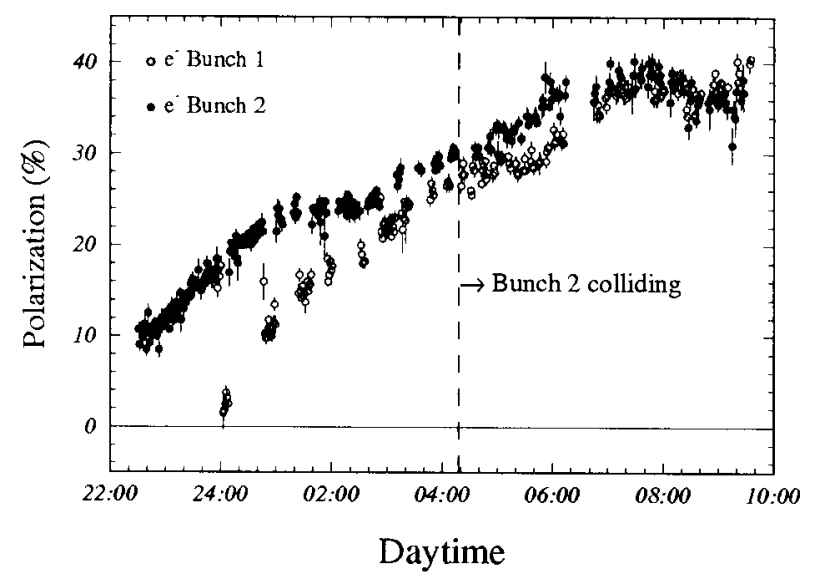

Figure. 3. Polarization level during third experiment

- the separators for the ${ }^{\circ}$ Pretzel scheme $^{\mathrm{o}}$, because the bunches did not meet in the arcs

The polarization level saturated at around $25 \%$ after applying the technique of deterministic harmonic spin matching.

Some time was then spend to further increase the polarization level by adopting a new method of educated harmonic spin matching ${ }^{\mathbf{0}}$ [6] [12], leading to a polarization level of more than $30 \%$. At that time electron bunch 2 and positron bunch 1 were brought into collision at interaction point 6 . The educated harmonic spin matching was pursued with beams in collision and a polarization level of $40 \%$ was obtained for both colliding and non colliding bunches. The luminosity was $1.0-1.5$ $\times 10^{30} \mathrm{~cm}^{-2} \mathrm{~s}^{-1}$ and the linear beam-beam tune shift was 0.04 .

\section{ESTIMATION OF BEAM-BEAM DEPOLARIZATION}

The asymptotic value for the polarization can be written as :

$$
P(t \rightarrow \infty)=\frac{0.924}{1+\left(\frac{\tau_{p}}{\tau_{d}}\right)_{s b}+\left(\frac{\tau_{p}}{\tau_{d}}\right)_{b b}}
$$

$\left(\tau_{p} / \tau_{d}\right)_{s b}$ includes all depolarizing effects in the presence of only one beam, $\left(\tau_{p} / \tau_{d}\right)_{b b}$ all depolarizing effects from the beambeam interaction.

During the third experiment the polarization levels of the colliding and the not colliding bunch were about the same. We can estimate the beam-beam depolarization since the polarization level of the non colliding bunch was $40 \%$ and since the difference with the colliding bunch did not exceed $4 \%$. Using the equation above this yields an equilibrium polarization level due to the beam-beam interaction of about $74 \%$, if no single beam depolarizing effects are present.

\section{SUMMARY}

The experimental study is far from being complete. We summarize our observations :

- In one experiment the beams were colliding at four points. The polarization level was limited to less than $10 \%$. No in- crease of the electron vertical beam size was observed. The vertical positron beam size increased by about $30 \%$.

- With the beam colliding at one point, a polarization level of $40 \%$ was achieved. The polarization level was about the same for one colliding and one non colliding bunch.

- It was observed that the polarization level depends critically on the synchrotron tune : when $Q_{s}$ was changed by 0.005 , the polarization strongly decreased.

- In one experiment with the beams colliding at four points at an energy of $45.60 \mathrm{GeV}$ with a linear beam-beam tune shift of $0.015 / \mathrm{IP}$ the depolarization was strong. In the last experiment performed at an energy of $44.71 \mathrm{GeV}$ the polarization level was $40 \%$ with a linear beam-beam tune shift of about $0.04 /$ IP. This indicates, that the beam-beam depolarization does not scale with the linear beam-beam tune shift at one crossing point. Other parameters as spin tune and synchrotron tune are also of importance.

The experiments presented here demonstrate that transverse polarization can be maintained in the presence of beam-beam effects, provided that the operational parameters are well selected. The results also show, that experiments with longitudinal polarized beams at least at one collision point are not prohibited by depolarizing beam-beam effects. However it should be kept in mind, that the beam-beam depolarizion with transverse and longitudinal polarized beams might be different.

\section{References}

[1] L.Arnaudon et al., Accurate determination of the LEP beam energy by resonant depolarization, CERN SL/94-71 (BI)

[2] G.Alexander et al., -Proposal- A Test of Longitudinal Spin Polarization at LEP, CERN/LEPC94-8, May 1994

[3] R.Assmann et al., Lepton Beam Polarization at LEP, Proc. Conf. High Energy Physics with Polarized Beams, Bloomington, Indiana, 1994

[4] K.Steffen, DESY PET82 (1982) and A.Blondel, LEP Note 629 (1990)

[5] R.Rossmanith, R.Schmidt, NIM A236(1985), 231-248, 1985

[6] R.Assmann, Transversale Spin-Polarization und ihre Anwendung fiùr Prikazisionsmessungen bei LEP, Doctoral Thesis, University Mürnchen

[7] K.Cornelis, Tail production due to beam-beam, Presentation 17, Proc. of the 3rd workshop on LEP Performance, CERN SL/93-19 (DI)

[8] R.Schmidt, Polarisation am Speicherring PETRA, Thesis University Hamburg, DESY Internal Report M 82/20, September 1982

[9] D.Barber, these proceedings

[10] see also : M.Placidi, Transverse Polarization at LEP, Proc. 5th Workshop on LEP Performance, Chamonix, CERN SL/95-08(DI)

[11] R.Bailey et al. PAC 1993, Washington, DC, USA, 17 - 20 May 1993

[12] A.Blondel, to be published 